Gut and Liver, Vol. 12, No. 5, September 2018, pp. 555-561

\title{
Measuring Intrahepatic Vascular Changes Using Contrast-Enhanced Ultrasonography to Predict the Prognosis of Alcoholic Hepatitis Combined with Cirrhosis: A Prospective Pilot Study
}

\author{
Min Sun Park ${ }^{1}$, Soonchang Hong ${ }^{2}$, Yoo Li Lim ${ }^{1}$, Seong Hee Kang ${ }^{1,3}$, Soon Koo Baik ${ }^{1,3}$, and Moon Young Kim ${ }^{1,3}$ \\ Departments of ${ }^{1}$ Internal Medicine, ${ }^{2}$ Thoracic and Cardiovascular Surgery, and ${ }^{3}$ Cell Therapy and Tissue Engineering; Yonsei University Wonju \\ College of Medicine, Wonju, Korea
}

Background/Aims: Acute hepatic dysfunction combined with alcoholic hepatitis (AH) in alcoholic cirrhosis is related to hepatic hypo-perfusion secondary to intrahepatic necroinflammation, neoangiogenesis, and shunt. The hepatic vein arrival time (HVAT) assessed by microbubble contrastenhanced ultrasonography (CEUS) is closely correlated with the severity of intrahepatic changes. We investigated the usefulness of HVAT to predict short-term mortality of AH in cirrhosis. Methods: Thirty-nine patients with alcoholic cirrhosis (27 males) and AH were prospectively enrolled. HVAT study was performed within 3 days after admission using ultrasonic contrast (SonoVue ${ }^{\circledR}$ ). The primary outcome was 12 week mortality. Results: Twelve-week mortality developed in nine patients. HVAT was significantly different between the mortality and survival groups $(9.3 \pm 2.0$ seconds vs $12.6 \pm 3.5$ seconds, $p=0.002$ ). The odds ratio of a shortened HVAT for 12-week mortality was 1.481 (95\% confidence interval, 1.050-2.090; $p=0.025)$. The area under the receiver operating characteristic curve of HVAT for 12-week mortality was $0.787(p=0.010)$. The combination of MDF and HVAT $\geq 11.0$ seconds resulted in an $87.5 \%$ survival rate even if the MDF score $\geq 32$; however, HVAT $<11.0$ seconds was related with mortality despite a MDF score $<32$. Conclusions: HVAT using microbubble CEUS could be a useful additional index to predict short-term mortality in patients with $\mathrm{AH}$ and cirrhosis. (Gut Liver 2018;12:555-561)

Key Words: Hepatitis, alcoholic; Liver cirrhosis, alcoholic; Hepatic veins; Ultrasonography, contrast-enhanced

\section{INTRODUCTION}

Alcoholic liver disease is one of leading cause of chronic liver disease and progression to end-stage liver disease. ${ }^{1}$ Alcoholic hepatitis (AH) is a phenotype of alcoholic liver disease and severe cases show high short-term mortality of $40 \%$ to $50 \% .{ }^{2,3}$ Cirrhosis is a pathological diagnosis characterized by diffuse fibrosis, severe disruption of the intrahepatic vascular system with neoangiogenesis and formation of a shunt resulting in portal hypertension and liver failure. ${ }^{4,5}$ Acute hepatic dysfunction combined with $\mathrm{AH}$ caused by binge drinking in patients with alcoholic cirrhosis is a common cause of acute on chronic liver failure and a hyper-acute poor prognosis. ${ }^{6}$ The poor prognosis is closely related to intrahepatic histological changes, including acute necroinflammation, secondary intrahepatic vascular disarrangement, and hepatic hypo-perfusion.

Several scoring systems have been used to predict survival of patients with AH, including Maddrey's Discriminant Function (MDF) score, ${ }^{7}$ the Model for End-Stage Liver Disease (MELD) score, the Glasgow Alcoholic Hepatitis score, age, serum bilirubin, international normalized ratio of prothrombin time and serum creatinine, ${ }^{10}$ the hepatic venous pressure gradient ${ }^{11}$ and the Lille model. ${ }^{12} \mathrm{MDF}$ is one of the most commonly used systems and when MDF is $\geq 32$ it is generally defined as severe alcoholic hepatitis (SAH). The treatment outcome of AH using prednisolone or pentoxifylline is relatively poor and is actually a clinically urgent situation that requires an early decision for liver transplantation (LT) before or regardless of starting treatment. ${ }^{13}$ These scoring systems have limitations because they include some serological components that do not properly represent the

Correspondence to: Moon Young Kim

Division of Gastroenterology and Hepatology, Department of Internal Medicine, Wonju Severance Christian Hospital, Yonsei University Wonju College of Medicine, 20 Ilsan-ro, Wonju 26426, Korea

Tel: +82-33-741-1229, Fax: +82-33-741-0950, E-mail: drkimmy@yonsei.ac.kr

Received on July 27, 2017. Revised on November 27, 2017. Accepted on December 13, 2017. Published online April 27, 2018

pISSN 1976-2283 eISSN 2005-1212 https://doi.org/10.5009/gnl17342

Min Sun Park and Soonchang Hong contributed equally to this work as first authors.

@ This is an Open Access article distributed under the terms of the Creative Commons Attribution Non-Commercial License (http://creativecommons.org/licenses/by-nc/4.0) which permits unrestricted non-commercial use, distribution, and reproduction in any medium, provided the original work is properly cited. 
rapid changes in disease severity and, particularly, the acute intrahepatic histological vascular changes in the early stage.

The hepatic vein arrival time (HVAT), as assessed by microbubble contrast-enhanced ultrasonography (CEUS), is the time (in second) taken for microbubble contrast agent to arrive at the hepatic vein (HV) after intravenous injection. It is a safe noninvasive method to estimate the severity and intrahepatic histological changes, particularly intrahepatic vascular changes. ${ }^{14-16}$ Therefore, we investigated the possibility of using HVAT as an additional prognostic index representing immediate intrahepatic histological changes to predict short-term mortality of patients with cirrhotic AH.

\section{MATERIALS AND METHODS}

\section{Study population}

Patients between 20 and 70 years of age with underlying cirrhosis and $\mathrm{AH}$ related with binge drinking alcohol who visited the Wonju Severance Christian Hospital, Yonsei University Wonju College of Medicine from March 2015 to February 2016 were considered eligible for this prospective study. The inclusion criteria were: (1) excessive alcohol consumption within 2 months (>60 g/day for males and $40 \mathrm{~g} /$ day for females); (2) rapid deterioration of liver function during the past 2 months; and (3) aspartate/alanine aminotransferase (AST/ALT) ratio greater than 2 with an AST level >40 IU/L. Exclusion criteria were: (1) those with liver disease not related to alcohol consumption (i.e., viral hepatitis, autoimmune hepatitis, and drug-induced liver injury) or hepatocellular carcinoma; and (2) those receiving pentoxifylline and/or corticosteroids before baseline HVAT study was performed. (3) In addition, patients who have already combined with infection and sepsis were also excluded. Patients who did not provide informed consent to participate were also excluded from the study. Thus, among 89 patients who were included in the initial screening, 50 patients were excluded, and
39 patients were finally enrolled and completed the laboratory tests, HVAT measurements, and a 12-week follow-up with appropriate medical therapies including steroid and pentoxifylline (Fig. 1).

\section{Study design and endpoint}

Baseline clinical data, Child-Pugh score, MDF, MELD, and HVAT were measured simultaneously on the same day within 3 days of admission. MDF was calculated with the equation: $\mathrm{MDF}=(4.6 \times$ [patient's prothrombin time in second-control's prothrombin time in second]+patient's serum bilirubin level [mg/ dL]). The MELD score was also calculated using an automatic calculating program at a website. After enrollment, the patients were followed up with or without special treatment, such as glucocorticoid or pentoxifylline, depending on the clinical indication. The primary endpoint was liver-related 12-week mortality, and the predictive values of each clinical scoring systems were evaluated.

This prospective study was approved by the Institutional Review Board of Wonju Severance Hospital (IRB No. CR211016) and performed in accordance with the principles of the Declaration of Helsinki. The informed consents were obtained.

\section{Measurement of hepatic vein arrival time}

All enrolled patients underwent measurement of HVAT using CEUS within 3 days of admission. After an overnight fast, all CEUS procedures were performed by an examiner (M.Y.K.) using a $2.5-\mu \mathrm{m}$ (range, 2 to $8 \mu \mathrm{m}$ ) second-generation sulfur hexafluoride microbubble-based contrast agent (SonoVue ${ }^{\circledR}$; Bracco SpA, Milan, Italy) according to a method described previously. ${ }^{15}$ The agent was injected into an antecubital vein, and signals were recorded from the right or middle hepatic veins for analysis. HVAT was calculated as the time interval from injection to the second point on the curve representing the signal intensity that exceeded the baseline intensity by $10 \%$ (Fig. 2). The HVAT cut-
50 Exclusion

31 Non-alcohol related liver disease

3 Hepatocellular carcinoma

16 Corticosteroid or pentoxifylline treatment
39 Final inclusion\&

HVAT measurement

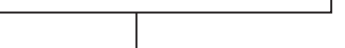

12 Weeks follow-up

30 Survival

9 Mortality
Fig. 1. Study design and flow chart HVAT, hepatic vein arrival time. 
off value (11.0 seconds) was determined according to Youden index based on receiver operating characteristic (ROC) curve analysis.

\section{Statistical analysis}

Continuous variables are presented as mean \pm standard deviation. Categorical variables are presented as counts and propor- tions. Group comparisons of continuous variables were conducted with the independent t-test and the Mann-Whitney Utest, as appropriate. Categorical variables were compared with the chi-square test. Pearson's correlation analysis was used for normally distributed variables and Spearman's rank-correlation coefficient analysis was performed for non-normally distributed data, as appropriate. To assess the performance of HVAT in pre-
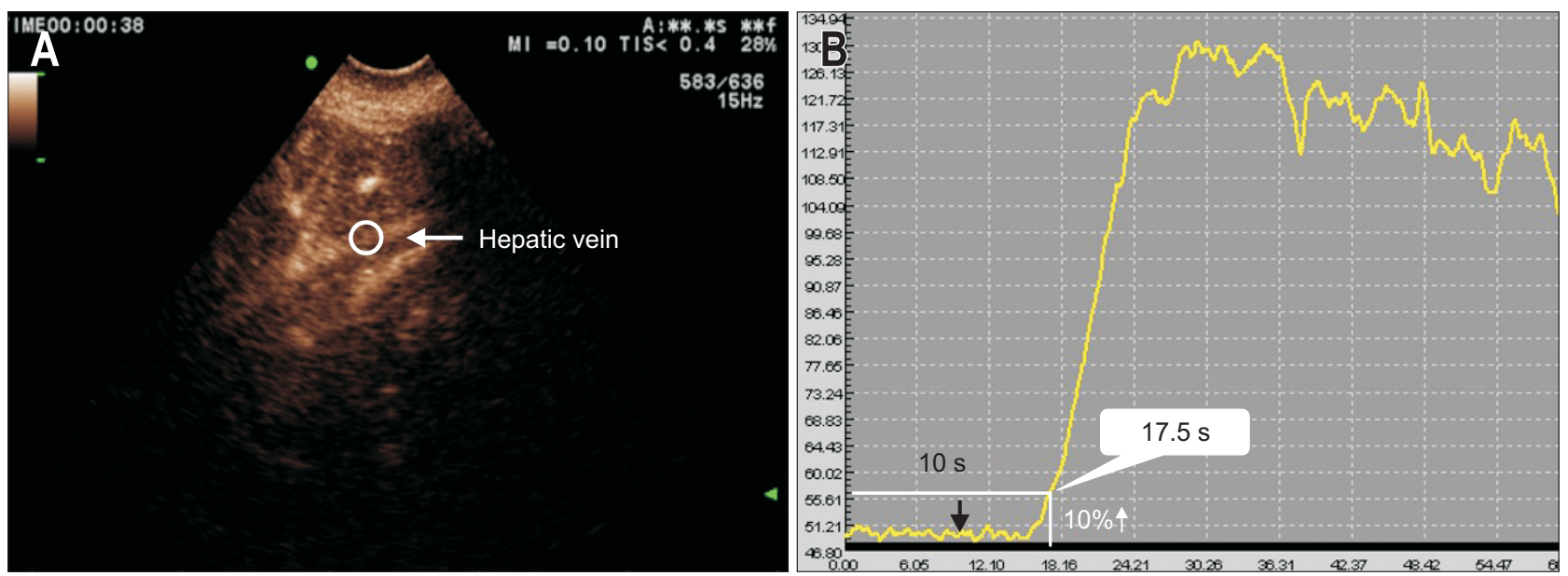

Fig. 2. Hepatic vein arrival time (HVAT) measurement using microbubble contrast enhanced ultrasonography (CEUS). (A) Detection of hepatic vein (HV) enhancement. After contrast injection at 10 seconds of lead time, HV enhancement with microbubble contrast agent was detected (white circle is region of interest [ROI] to measure time intensity curves [TICs]). (B) TICs for HV enhancement intensity were drawn and HVAT was calculated as the time (in seconds) from injection to a sustained signal increase in the TIC to over 10\% above baseline intensity point (the 10-second lead time [black arrow] should be subtracted from the measured time point [e.g., 17.5-10.0 seconds=7.5 seconds]).

Table 1. General Characteristics

\begin{tabular}{|c|c|c|c|c|}
\hline Characteristic & Total population $(\mathrm{n}=39)$ & Survival $(\mathrm{n}=30)$ & Mortality (n=9) & p-value* \\
\hline Male sex & 27 (69.0) & $22(73.3)$ & $5(55.6)$ & 0.416 \\
\hline Age, yr & $50.0(35.0-69.0)$ & $51.0(35.0-68.0)$ & $46.0(42.0-69.0)$ & 0.921 \\
\hline Child-Pugh score & $9.0(8.0-11.0)$ & $9.0(5.0-10.0)$ & $10.0(7.0-13.0)$ & 0.007 \\
\hline MELD score & $19.5(6.0-40.0)$ & $16.0(6.0-28.0)$ & $27.0(7.0-40.0)$ & $<0.001$ \\
\hline MDF score & $49.0(1.6-113.9)$ & $32.2(1.6-80.0)$ & $78.2(58.4-113.9)$ & $<0.001$ \\
\hline HVAT, s & $11.0(6.4-19.4)$ & $12.2(7.1-19.4)$ & $8.7(6.4-16.8)$ & 0.002 \\
\hline Total bilirubin, $\mathrm{mg} / \mathrm{dL}$ & $8.7(0.8-54.6)$ & $4.8(0.8-28.9)$ & $21.9(1.1-54.6)$ & 0.001 \\
\hline AST, U/L & $165.5(50.0-2,185.0)$ & $296.0(50.0-2,185.0)$ & $150.0(61.0-300.0)$ & 0.005 \\
\hline ALT, U/L & $85.5(17.0-2,635.0)$ & $256.0(20.0-2,635.0)$ & $37.0(17.0-252.0)$ & 0.005 \\
\hline Albumin, g/dL & $3.1(2.0-4.4)$ & $3.3(2.2-4.4)$ & $3.0(2.0-3.6)$ & 0.175 \\
\hline INR & $1.5(0.9-2.7)$ & $1.4(0.9-2.3)$ & $2.0(1.0-2.7)$ & $<0.001$ \\
\hline WBC, $10^{9} / \mathrm{L}$ & $5,765.0(1,730.0-32,490.0)$ & $5,500.0(1,730-32,490)$ & $6,750.0(4,150.0-19,000.0)$ & 0.018 \\
\hline $\mathrm{Hb}, \mathrm{g} / \mathrm{dL}$ & $11.8(8.3-17.3)$ & $12.1(8.5-17.3)$ & $11.0(8.3-14.4)$ & 0.130 \\
\hline Platelet, $10^{9} / \mathrm{L}$ & $106.0(25.0-443.0)$ & $110.0(25.0-443.0)$ & $80.0(25.0-295.0)$ & 0.817 \\
\hline CRP, mg/dL & $0.9(0.1-7.8)$ & $0.6(0.1-7.8)$ & $2.0(0.3-4.4)$ & 0.173 \\
\hline $\mathrm{Cr}, \mathrm{mg} / \mathrm{dL}$ & $0.7(0.2-3.5)$ & $0.6(0.2-1.5)$ & $0.7(0.2-3.5)$ & 0.305 \\
\hline $\mathrm{Na}, \mathrm{mmol} / \mathrm{L}$ & $137.0(117.0-144.0)$ & $138.0(117.0-144.0)$ & $137.0(125.0-139.0)$ & 0.360 \\
\hline
\end{tabular}

Data are presented as number (\%) or median (range).

MELD, Model for End-Stage Liver Disease; MDF, Maddrey's Discriminant Function; HVAT, hepatic vein arrival time; AST, aspartate aminotransferase; ALT, alanine aminotransferase; INR, international normalized ratio; WBC, white blood cell; Hb, hemoglobin; CRP, C-reactive protein.

${ }^{*}$ Statistical comparison between survival and mortality. 
dicting mortality, ROC curves and the area under the ROC curve (AUROC) were calculated. Odds ratios of the factors predicting 12-week mortality were calculated using binary logistic regression analysis. A p-value $<0.05$ was considered significant. The statistical analysis was performed with IBM SPSS version 20.0 software (IBM Corp., Armonk, NY, USA).

\section{RESULTS}

The baseline general characteristics of study populations are summarized in Table 1 . Among the 39 included patients, nine patients died within 12 weeks. The causes of death were gastrointestinal bleeding (one patient), spontaneous bacterial peritonitis (one patient), sepsis (two patients), hepatic failure (five patients). As expected, the indices suggesting hepatic dysfunction, such as the Child-Pugh score, MDF, and MELD score, were higher and HVAT was significantly shorter in the mortality group. Leukocytosis was also prominent in the mortality group compared to the surviving group (Table 1).

\section{Traditional predictive scoring systems related to mortality}

Significant relationships were observed between mortality and the clinical scores. The increases in the Child-Pugh, the MELD, and MDF scores indicated an increased risk for 12-week mortality (odds ratios $=2.469,1.434$, and 1.071 respectively) (Table 2). The AUROC values for 12-week mortality with the ChildPugh score, MELD score, and MDF score were $0.804(\mathrm{p}=0.006)$, $0.915(\mathrm{p}<0.001)$, and 0.915 ( $\mathrm{p}<0.001)$, respectively.

\section{Hepatic vein arrival time and mortality}

HVAT was also significantly related with 12-week mortality. HVAT was significantly lower in the mortality group than that in the survival group $(9.3 \pm 2.0$ seconds vs $12.6 \pm 3.5$ seconds, $\mathrm{p}=0.002$ ) (Table 1). The odds ratio of HVAT for 12-week mortality was 0.675 (95\% confidence interval [CI], 0.478 to 0.953) (Table 2). In other words, when 1 second HVAT was shortened, the mortality risk increased 48.1\% (odds ratio, 1.481; 95\% CI, 1.050 to 2.090, $\mathrm{p}=0.025$ ). The AUROC of HVAT for 12 -week mortality was $0.787(p=0.010)$, it was relatively lower than conventional predictive scoring systems. The sensitivity, specificity, positive predictive value, and negative predictive values, accuracy according to the HVAT cutoff value of $<11.0$ seconds were $88.9 \%$, $66.7 \%, 44.4 \%, 95.2 \%$, and 0.72 respectively. Significant negative correlations were observed between HVAT and Child-Pugh score $(R=-0.421, p=0.008)$, MELD score $(R=-0.532, p<0.001)$, and MDF score $(\mathrm{R}=-0.547, \mathrm{p}<0.001)$.

In a multivariate analysis based on univariate analysis, HVAT showed significant odds ratio for the prediction of 12-week mortality in each model including Child-Pugh score (model 1), MELD score (model 2) and MDF (model 3) (Table 2).

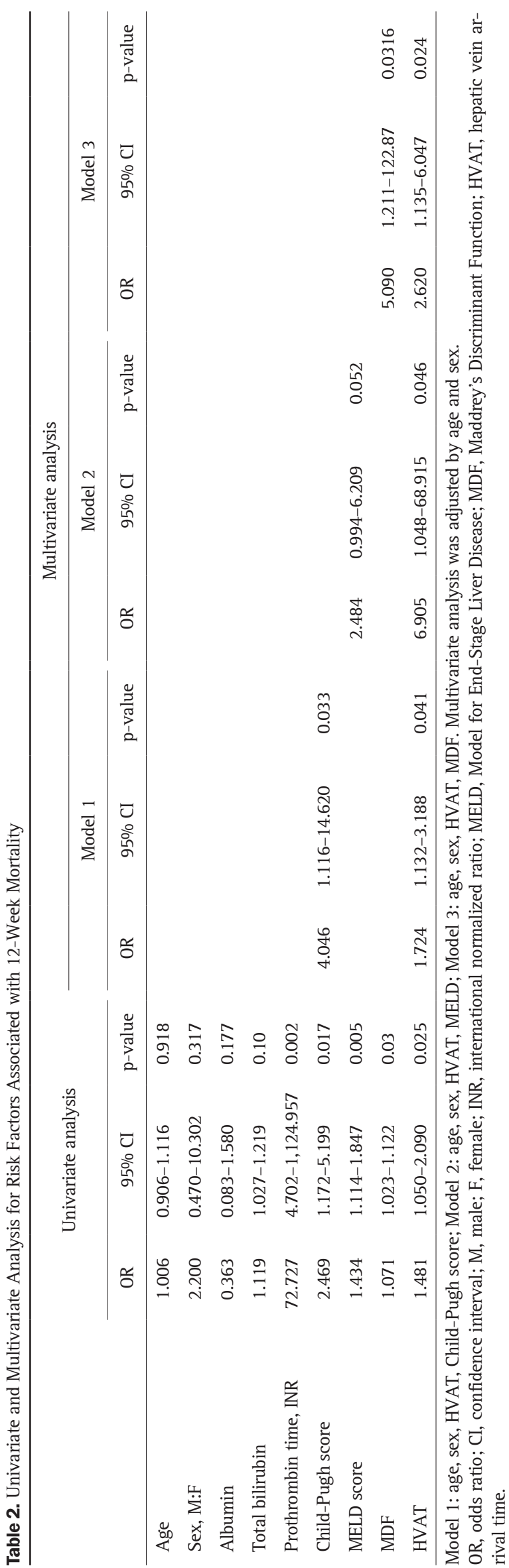




\section{The additional predictive value of hepatic vein arrival time for mortality}

\section{1) Additional predictive value of HVAT on MDF}

Among patients who showed baseline MDF <32, just one death occurred who simultaneously showed HVAT $<11.0$ seconds. However, no mortality developed in patients who had a HVAT $\geq 11.0$ seconds. Eight mortalities occurred among 21 patients with baseline MDF $\geq 32$, and most (7/8, 87.5\%) had a HVAT $<11.0$ seconds. In contrast, the survival rate was $87.5 \%$ when HVAT $\geq 11.0$ seconds, even though the patient initially had a MDF $\geq 32$ (Fig. 3).

\section{2) Additional predictive value of HVAT on MELD}

One mortality was combined with a HVAT $<11.0$ seconds in a patient with baseline MELD $<21$. However, no mortality occurred in patients who showed HVAT $\geq 11.0$ seconds in the MELD $<21$ group. Eight deaths occurred in 18 patients with MELD $\geq 21$, and seven of the eight mortalities (87.5\%) presented with a HVAT $<11.0$ seconds. In contrast, $83.3 \%$ of patients who had HVAT $\geq 11.0$ seconds survived, even though they initially presented with a MELD $\geq 21$ (Fig. 4).

\section{DISCUSSION}

A number of trials have tried to develop an optimal treatment for AH but only glucocorticoids and pentoxifylline have been accepted as treatment. ${ }^{17,18}$ However, their effect does not seem sufficient and their survival benefit has been controversial in the past several decades. ${ }^{13}$ Therefore, there is an need for early prediction of a poor prognosis and making a decision for early preparation for LT regardless of the conventional treatment. In general, a MDF value $\geq 32$ has been accepted as SAH and presents a very poor prognosis, with mortality of $20 \%$ to $30 \%$ within 1 month after development and 30\% to 40\% within 6 months. ${ }^{7}$ Although the MDF has been helpful in predicting the prognosis and decision for treatment, there still has been some discrepancies and difficulties predicting mortality in clinical practice, so several other prognosis scoring systems have been tried. $^{8-12}$

$\mathrm{AH}$ is accompanied by hepatic parenchymal necroinflammation, destruction, and neovascularization; these findings are directly related with clinical outcome and the poor prognosis. If AH develops in a patient with underlying cirrhosis, the clinical outcome might be more dismal. Thus, estimating histological severity is essential in the prognostic scoring system; however,
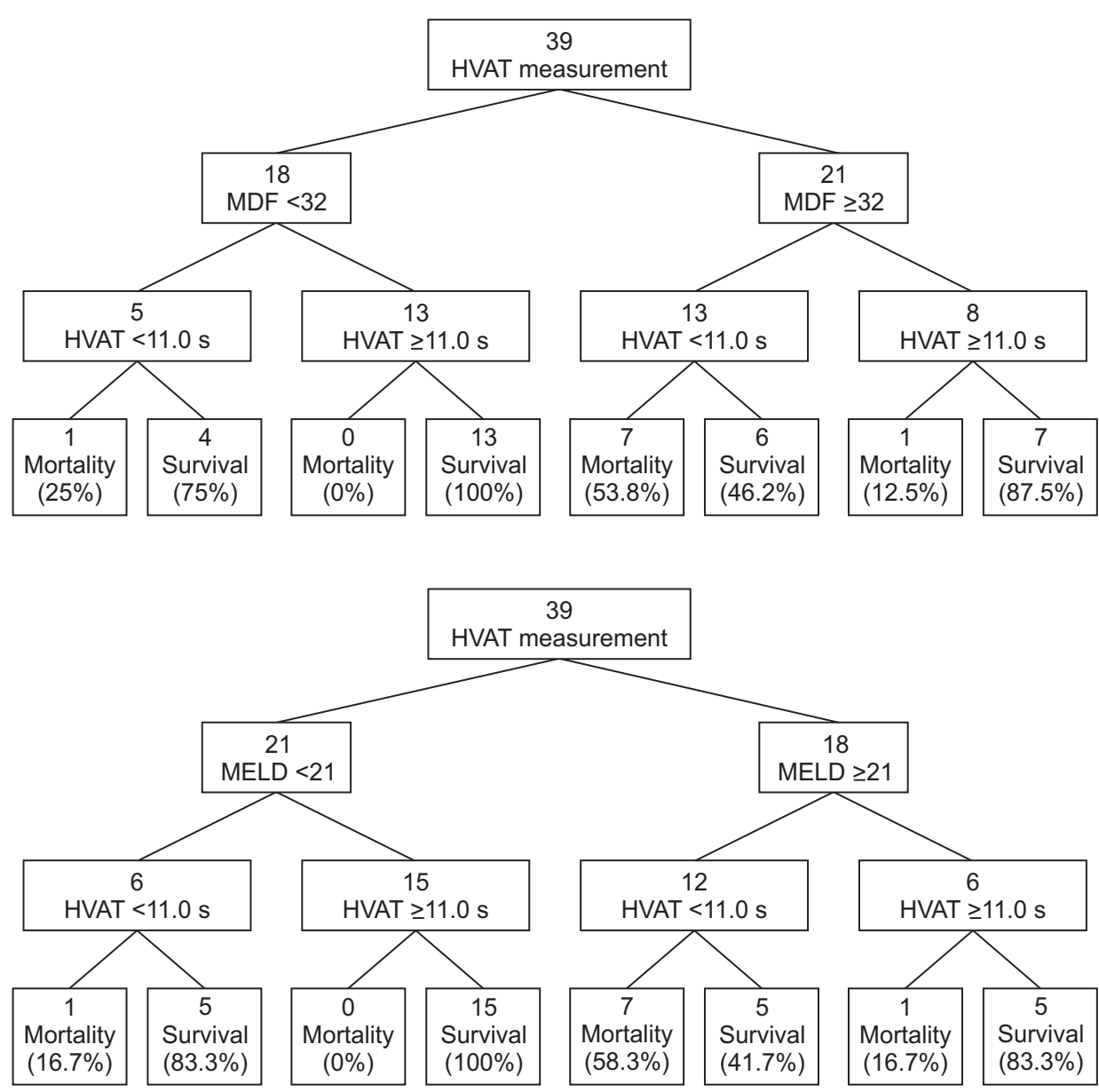

Fig. 3. The additional benefit of using hepatic vein arrival time (HVAT) to make a prognosis based on a Maddrey's Discriminant Function (MDF) score. Among patients with a baseline MDF of $<32$, only one mortality developed with a simultaneous HVAT of $<11.0$ seconds. However, when HVAT was $\geq 11.0$ seconds, the survival rate was $87.5 \%$, although the baseline MDF was $\geq 32$.
Fig. 4. The additional benefit of using hepatic vein arrival time (HVAT) to make a prognosis based on Model for End-Stage Liver Disease (MELD) score. Only one mortality occurred in patients with a HVAT of $<11.0$ seconds and a MELD of $<21$. In contrast, $83.3 \%$ of patients with a HVAT of $\geq 11.0$ seconds survived despite initially presenting a MELD of $\geq 21$. 
no scoring system includes a histological component. A liver biopsy is not usually recommended for a patient with $\mathrm{AH}$ in clinical practice because of its invasiveness and the risk of coagulopathy. Therefore, developing a noninvasive histological index would improve clinical practice.

The HVAT is the time (in second) taken for the microbubble contrast agent to arrive at the HV after passing through the systemic and intrahepatic circulation. The microbubble ultrasonography contrast agent normally flows into the liver by two pathways, such as the hepatic artery and the splanchnic circulation-portal vein. After arriving at the liver, the contrast flows through the intrahepatic sinusoids and reaches the HV. The severity of hepatic fibrosis in chronic liver disease is strongly correlated with early enhancement of the HV and shortening of HVAT, ${ }^{14,19}$ and there are sufficient data on the relationship between HVAT and hepatic vein transit time and portal hypertension. ${ }^{15,16,20}$ Shortening the HVAT in patients with cirrhosis is secondary to intrahepatic hemodynamic changes, such as arteriovenous shunting, unfavorable neoangiogenesis, and sinusoidal capillarization. ${ }^{14,19-22}$ In these conditions, contrast agent bypasses diffusion into a sinusoid and flows directly into the central vein or branch of the HV, so arrival time to the HV is shortened. Shortening of HVAT also reflects deterioration of hepatic function, which is aggravated by impaired liver-tissue oxygenation and hepatic perfusion secondary to intrahepatic histological changes. ${ }^{23,24}$ These findings can be more severe when $\mathrm{AH}$ is combined with cirrhosis.

In the present study, we evaluated the usefulness of HVAT as a noninvasive index reflecting the intrahepatic histological state in AH. HVAT showed good prognostic value, and a 1 second shortening of HVAT increased the 12-week mortality risk by 48.1\%. In particular, the 11.0 seconds HVAT cutoff value is a very unique finding with high sensitivity (88.9\%) and negative predictive value $(95.2 \%)$ for 12 -week mortality. This result suggests that HVAT $<11.0$ seconds can be a useful index to detect high mortality risk patients and to rule out patients who have a relatively low risk of mortality. This is a very important point because classifying patients early who need LT before conventional treatment is critical in clinical practice in the absence of a definitive reliable treatment. Although HVAT did not show superiority in the AUROC compared with traditional prognostic scoring systems to predict 12-week short-term mortality, it was still very useful to combine with the traditional scoring systems. According to the MDF and HVAT combined model, 87.5\% of patients who presented with HAVT $\geq 11.0$ seconds survived even though their MDF score was $\geq 32$. In contrast, one of five patients with HVAT $<11.0$ seconds and MDF $<32$ died (Fig. 3). Similar findings were observed when using the combined HVAT and MELD model (Fig. 4). These results suggest that HVAT can provide additional prognostic accuracy when combined with a traditional scoring system as a histological severity based index in patients with AH. This combined model can be useful for early LT before the development of multi-organ failure including hepatorenal syndrome and acute-on chronic liver failure, especially in a patient with SAH.

This study had some limitations. This was a pilot study, and the small sample size limited clinical adaptation. However, as mentioned above, this study documents the possibility of an additional effect of HVAT, and a future large-sized well-designed prospective study is warranted to validate the findings. No histological findings were documented through liver biopsy. However, sufficient data are available for the correlation between histological and CEUS findings and the ethics issue did not permit a highly invasive procedure in patients with $\mathrm{AH}$ and a high risk for mortality.

In conclusion, this pilot study prospectively evaluated the clinical usefulness of HVAT. The assessment of intrahepatic histological changes especially vascular changes in AH could be benefit to predict short-term mortality. HVAT has been known to have relation with the acute and chronic histological changes, especially vascular changes including neovascularization, and shunting. The present pilot study showed that the combined interpretation of HVAT and conventional scoring systems can be more useful in the prediction of mortality of SAH, especially to detect high risk patients early and exclude low risk patients with SAH.

\section{CONFLICTS OF INTEREST}

No potential conflict of interest relevant to this article was reported.

\section{ACKNOWLEDGEMENTS}

This research was supported by the Basic Science Research Program through the National Research Foundation of Korea (NRF) funded by the Ministry of Science, ICT, \& Future Planning (2017R1A2B4009199).

\section{REFERENCES}

1. Kim WR, Brown RS Jr, Terrault NA, El-Serag H. Burden of liver disease in the United States: summary of a workshop. Hepatology 2002;36:227-242.

2. Lucey MR, Mathurin P, Morgan TR. Alcoholic hepatitis. N Engl J Med 2009;360:2758-2769.

3. Mathurin P, Duchatelle V, Ramond MJ, et al. Survival and prognostic factors in patients with severe alcoholic hepatitis treated with prednisolone. Gastroenterology 1996;110:1847-1853.

4. Schuppan D, Afdhal NH. Liver cirrhosis. Lancet 2008;371:838851.

5. Jang YO, Jun BG, Baik SK, Kim MY, Kwon SO. Inhibition of hepatic stellate cells by bone marrow-derived mesenchymal stem cells in hepatic fibrosis. Clin Mol Hepatol 2015;21:141-149. 
6. Hernaez R, Solà E, Moreau R, Ginès P. Acute-on-chronic liver failure: an update. Gut 2017;66:541-553.

7. Maddrey WC, Boitnott JK, Bedine MS, Weber FL Jr, Mezey E, White RI Jr. Corticosteroid therapy of alcoholic hepatitis. Gastroenterology 1978;75:193-199.

8. Dunn W, Jamil LH, Brown LS, et al. MELD accurately predicts mortality in patients with alcoholic hepatitis. Hepatology 2005;41:353-358.

9. Forrest EH, Evans CD, Stewart S, et al. Analysis of factors predictive of mortality in alcoholic hepatitis and derivation and validation of the Glasgow alcoholic hepatitis score. Gut 2005;54:11741179.

10. Dominguez M, Rincón D, Abraldes JG, et al. A new scoring system for prognostic stratification of patients with alcoholic hepatitis. Am J Gastroenterol 2008;103:2747-2756.

11. Rincon D, Lo Iacono 0, Ripoll C, et al. Prognostic value of hepatic venous pressure gradient for in-hospital mortality of patients with severe acute alcoholic hepatitis. Aliment Pharmacol Ther 2007;25:841-848.

12. Louvet A, Naveau S, Abdelnour M, et al. The Lille model: a new tool for therapeutic strategy in patients with severe alcoholic hepatitis treated with steroids. Hepatology 2007;45:1348-1354.

13. Thursz MR, Richardson P, Allison M, et al. Prednisolone or pentoxifylline for alcoholic hepatitis. N Engl J Med 2015;372:16191628.

14. Lim AK, Taylor-Robinson SD, Patel N, et al. Hepatic vein transit times using a microbubble agent can predict disease severity noninvasively in patients with hepatitis C. Gut 2005;54:128-133.

15. Kim MY, Suk KT, Baik SK, et al. Hepatic vein arrival time as assessed by contrast-enhanced ultrasonography is useful for the assessment of portal hypertension in compensated cirrhosis. Hepatology 2012;56:1053-1062.
16. Maruyama H, Shiha G, Yokosuka 0, et al. Non-invasive assessment of portal hypertension and liver fibrosis using contrastenhanced ultrasonography. Hepatol Int 2016;10:267-276.

17. O'Shea RS, Dasarathy S, McCullough AJ; Practice Guideline Committee of the American Association for the Study of Liver Diseases; Practice Parameters Committee of the American College of Gastroenterology. Alcoholic liver disease. Hepatology 2010;51:307-328.

18. European Association for the Study of Liver. EASL clinical practical guidelines: management of alcoholic liver disease. J Hepatol 2012;57:399-420.

19. Blomley MJ, Lim AK, Harvey CJ, et al. Liver microbubble transit time compared with histology and Child-Pugh score in diffuse liver disease: a cross sectional study. Gut 2003;52:1188-1193.

20. Jeong WK, Kim TY, Sohn JH, Kim Y, Kim J. Severe portal hypertension in cirrhosis: evaluation of perfusion parameters with contrast-enhanced ultrasonography. PLoS One 2015;10:e0121601.

21. Blomley MJ, Albrecht T, Cosgrove DO, et al. Liver vascular transit time analyzed with dynamic hepatic venography with bolus injections of an US contrast agent: early experience in seven patients with metastases. Radiology 1998;209:862-866.

22. Sugimoto H, Kaneko T, Hirota M, Tezel E, Nakao A. Earlier hepatic vein transit-time measured by contrast ultrasonography reflects intrahepatic hemodynamic changes accompanying cirrhosis. J Hepatol 2002;37:578-583.

23. Huet PM, Goresky CA, Villeneuve JP, Marleau D, Lough JO. Assessment of liver microcirculation in human cirrhosis. J Clin Invest 1982;70:1234-1244.

24. Berzigotti A, Nicolau C, Bellot P, et al. Evaluation of regional hepatic perfusion (RHP) by contrast-enhanced ultrasound in patients with cirrhosis. J Hepatol 2011;55:307-314. 